\title{
Encapsulation of Paramagnetic Chelates in Perfluorocarbon-loaded Fractal Nanoparticles Enables Modulation of Fluorine-19 and Proton Magnetic Resonance Imaging Signal
}

Margot Verbeelen ${ }^{1, \ddagger}$, Paul B. White ${ }^{2, \ddagger}$, Alexander H.J. Staal ${ }^{1}$, Edyta Swider-Cios ${ }^{1}$, Kimberley R. G. Cortenbach ${ }^{1}$, N. Koen van Riessen ${ }^{1}$, Cyril Cadiou $^{3}$, Françoise Chuburu ${ }^{3}$, Mangala Srinivas ${ }^{1,4}$, $5 \ddagger, \star$, Olga Koshkina ${ }^{1, \ddagger, *}$

${ }^{1}$ Department of Tumor Immunology, Radboud Institute for Molecular Life Sciences, Radboud University Medical Center, Nijmegen, The Netherlands 2Institute for Molecules and Materials, Radboud University, Nijmegen, The Netherlands

3/CMR Equipe Chimie de Coordination, Universite de Reims

${ }^{4}$ Cenya Imaging B.V., Amsterdam, The Netherlands

${ }^{5}$ Department of Cell Biology and Immunology, Wageningen University and Research, Wageningen, The Netherlands

\footnotetext{
Abstract

${ }^{19} \mathrm{~F}$ magnetic resonance imaging $\left({ }^{19} \mathrm{~F} \mathrm{MRI}\right)$ is an emerging technique for quantitative imaging of novel therapies, such as cellular therapies and theranostic nanocarriers. A modification of perfluorocarbon (PFC)-loaded, nanocarrier-based ${ }^{19} \mathrm{~F}$ MRI probes with paramagnetic chelates can enhance probe's functionality. Liquid PFC-loaded nanocarriers typically have a core-shell structure with PFC in the core due to the poor miscibility of PFC. However, paramagnetic relaxation enhancement acts only at a distance of a few angstroms. Thus, efficient modulation of ${ }^{19} \mathrm{~F}$ signal is possible only with fluorophilic PFC-soluble chelates. Such chelates, however, cannot interact with the surroundings of nanocarriers. Conversely, chelates on the surface typically affect only the aqueous environment but not the ${ }^{19} \mathrm{~F}$ signal.
} 
We show that the confinement of PFC in biodegradable polymeric nanoparticles with fractal structure enables modulation of longitudinal and transverse ${ }^{19} \mathrm{~F}$ relaxation, as well as proton signal, using non-fluorophilic paramagnetic chelates. We compared nanoparticles with fractal multicore versus conventional core-shell structure, where the PFC is encapsulated in the core(s) and the chelate in the surrounding polymeric matrix. Importantly, paramagnetic chelates affected both longitudinal and transverse ${ }^{19} \mathrm{~F}$ relaxation in fractal multicore nanoparticles, but not in core-shell nanocapsules. Both relaxation rates of ${ }^{19} \mathrm{~F}$ nucleus increased with an increasing concentration of the paramagnetic chelate. Moreover, as the polymeric matrix remained water-permeable, proton enhancement additionally was observed in MRI. In the future, the effects of fractal confinement could be combined with more effective paramagnetic chelates to develop multifunctional imaging probes, for example, for high-sensitivity ${ }^{19} \mathrm{~F}$ MRI combined with sensing.

Keywords: ${ }^{19} \mathrm{~F} \mathrm{MRI}$, paramagnetic relaxation enhancement, PLGA, perfluorocarbon, fractal nanoparticles, confinement

${ }^{19} \mathrm{~F}$ Magnetic Resonance Imaging ( $\left.{ }^{19} \mathrm{~F} \mathrm{MRI}\right)$ is an emerging technique which is in experimental clinical use. ${ }^{1-3}{ }^{19} \mathrm{~F} \mathrm{MRI}$ is able to unambiguously localize, and also quantify the imaging agent, without endogenous background, combined with anatomic proton imaging. 1, 2, 4, 5 These features make ${ }^{19} \mathrm{~F}$ MRI very powerful in a broad range of applications, particularly in tracking of cellular therapies or therapeutic nanocarriers, and for the design of activatable probes. ${ }^{2,3,6-9}$ Combination of liquid perfluorocarbons (PFC), and paramagnetic chelates in nanocarriers can lead to multifunctional imaging probes. Paramagnetic chelates on the surface of nanocarriers can be used to sense the biological environment of the probe. ${ }^{10-12}$ Conversely, fluorophilic chelates that enhance the longitudinal ${ }^{19} \mathrm{~F}$-relaxation rate increase the sensitivity of ${ }^{19} \mathrm{~F}$ MR signal. ${ }^{13-16}$ However, it remains difficult to develop nanosystems in which the paramagnetic chelate modulates both the longitudinal ${ }^{19} \mathrm{~F}$-relaxation and the proton signal. Here, we studied the effects of internal structure 
of polymeric nanoparticles on Paramagnetic Relaxation Enhancement (PRE), and showed that a confining fractal structure can be used for design of imaging agents with PRE of both ${ }^{19} \mathrm{~F}$-nucleus and proton.

PRE acts over very short distances in a range of angstroms, and decreases with the radius $\mathrm{r}^{6}$, as described by the Solomon-Bloembergen-Morgan equations. ${ }^{10,14,16,17}$ Hence, the paramagnetic chelate and the nucleus that should be modulated have to be very close to each other, in the range of angstroms. ${ }^{10,14,16}$ However, liquid PFC are both hydrophobic and lipophobic. ${ }^{18-20}$ As a result, common nanosized ${ }^{19} \mathrm{~F} \mathrm{MRI}$ imaging agents, are emulsions or core-shell capsules with a liquid PFC in the core that are stabilized either by a surfactant, polymer or silica shell.1, 2, 4, 5, 21, 22 The core sizes are usually at least several 10's of nanometers. ${ }^{1,2,4,5}$ As a result of the core-shell structure, currently, there are two strategies for the modification with paramagnetic chelates: (1) covalent attachment or encapsulation of the hydrophilic or hydrophobic chelates in the organic shell of the agent, ${ }^{12,23,24}$ and (2) encapsulation of fluorophilic chelates in the fluorous core. ${ }^{13-15,25}$

When a paramagnetic chelate is encapsulated or covalently bound to the shell, it typically affects only a small fraction of ${ }^{19} \mathrm{~F}$-nuclei that are close to the shell. ${ }^{23,}{ }^{24}$ Thus, only modest effects on ${ }^{19} \mathrm{~F}$ longitudinal relaxation rates can be achieved;23, 24 moreover, the signal can become inhomogeneous due to different relaxation rates of ${ }^{19} \mathrm{~F}$ nuclei, depending on their distance of the chelate. ${ }^{25}$ In spite of the low effect on ${ }^{19} \mathrm{~F}$-nucleus, this approach enables the modulation of protons in the surrounding medium and detection of other ions, making such probes promising for biosensing. ${ }^{12}$ Conversely, the fluorophilic chelates, as first introduced by the Ahrens-group, are soluble in the fluorous core of the imaging agents. ${ }^{13-15,25}$ As a result the distance between ${ }^{19} \mathrm{~F}$ nuclei and paramagnetic ions decreases, altering the longitudinal relaxation properties of ${ }^{19} \mathrm{~F}$ nuclei. Depending on the ion, this strategy can be used to increase the imaging sensitivity of the PFC-emulsions. ${ }^{13,14}$ However, in such emulsions the paramagnetic nuclei are isolated from the aqueous environment, making simultaneous modulation of the proton signal impossible. 
Our group has introduced polymeric PFC-loaded nanoparticles that display a structure different from conventional core-shell systems, namely the fractal multicore structure (Figure 1a).26, 27 These nanoparticles contain multiple small cores of perfluoro-15-crown-5 ether (PFCE) with a radius of $10-12 \mathrm{~nm}$ that are encapsulated in a matrix of biocompatible and biodegradable poly(lactic-co-glycolic acid) (PLGA) (Figure 1a, b). ${ }^{26}$ This structure is advantageous for in vivo and clinical use, as it results in about 15-fold faster clearance of PFCE compared to the PFCE emulsions. ${ }^{28-30}$ Conversely emulsions and other core-shell systems can display organ accumulation times up to several months. ${ }^{28,} 29$ Moreover, the use of a biocompatible PLGA allows for the encapsulation of compounds for multimodal imaging and therapy. ${ }^{26,}$, $30-33$ These nanoparticles can be produces at clinical grade and received approval for a clinical trial. Thus, combining these systems with paramagnetic probes could open up new opportunities in the development of probes for combined imaging, biosensing and delivery of therapeutics.

Here, we explored how the formation of fractal multicore structure and the resulting nanoconfinement of PFCE affects the PRE. Therefore, we used lipophilic gadolinium chelates which were encapsulated in the polymer matrix of the fractal nanoparticles. We compared their relaxation properties with core-shell nanocapsules. The changes of ${ }^{19} \mathrm{~F}$-relaxation in core-shell systems were minor, as expected. Conversely, in multicore nanoparticles, we detected enhancement of both longitudinal and transverse relaxation rates, depending on the concentration of encapsulated gadolinium. Furthermore, differently from conventional emulsions, the proton signal could be modulated as well. Overall, we demonstrate that the structural properties of the nanoparticles, and the resulting confinement effects can alter the MR properties. Thus, this approach can be adapted for the development of dual ${ }^{1} \mathrm{H} /{ }^{19} \mathrm{~F}$ MR imaging agents co-loaded with PFC and paramagnetic compounds for different applications, particularly biosensing and enhanced relaxation probes. 


\section{Results and discussion}

\section{Lipophilic chelates can be efficiently encapsulated in both fractal multicore}

nanoparticles and core-shell nanocapsules.

To study the effects of the structural properties on the MR properties of nanoparticles that coencapsulate gadolinium chelates and PFCE, we compared the behavior of fractal multicore PFCEPLGA nanoparticles with core-shell nanocapsules (Figure 1a). As gadolinium-agents we used two different hydrophobic gadolinium chelates that differ only in the length of a hydrophobic linker (Figure 1). ${ }^{34}$ The chelates are hydrophobic, but not fluorophilic, and therefore should remain in the hydrophobic PLGA matrix.

Both fractal multicore nanoparticles and core-shell nanocapsules can be produced in a miniemulsion formulation approach, ${ }^{26}$ adjusting the concentration of the lipophilic gadolinium chelate in the PLGA matrix. Here, we used different amounts of both lipophilic chelates, between 0 and $2.4 \mathrm{mg} \mathrm{Gd}$. A hydrophilic clinical contrast agent, gadoteridol, was used as an additional control (Prohance; $140 \mathrm{mg} \mathrm{Gd}$ ). After the synthesis, nanoparticles underwent an extensive purification to remove any free chelate.

The co-encapsulation of the gadolinium chelates did not affect the size of the resulting nanoparticles (Figure 1c,d, Tables S1 and S2). The cryogenic Scanning Electron Microscopy (cryoSEM) images of nanoparticles and capsules are shown in Figure 1c,d. All gadolinium-loaded multicore nanoparticles display the diameters around $200 \mathrm{~nm}$ and a monomodal size distribution with polydispersity indices (PDI) close to 0.1 , as shown by Dynamic Light Scattering (DLS, Table S1). The capsules were slightly smaller and had diameters of 150-180 nm (Table S2). Both size and PDI are similar to a control without gadolinium chelate, and the variation between different samples is within a typical batch-to-batch variation range. ${ }^{26}$ 


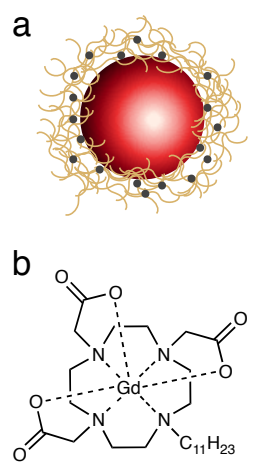

Gd_01

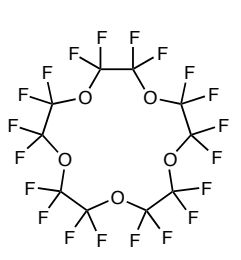

PFCE

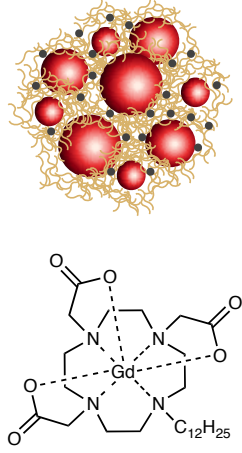

Gd_02
- PFCE

PLGA / PVA

- Gd-chelate
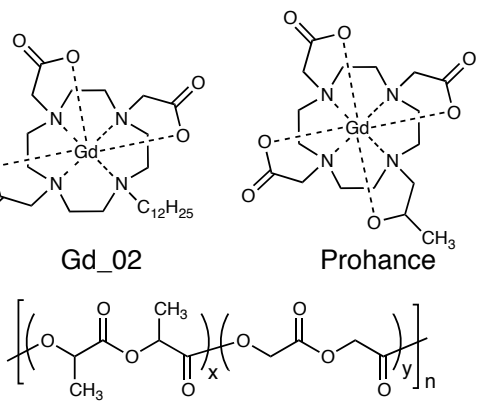

PLGA

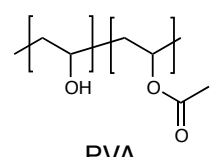

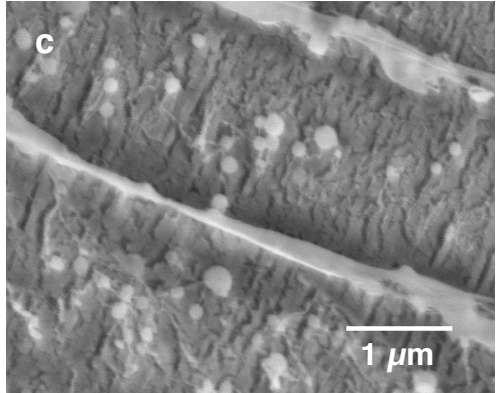

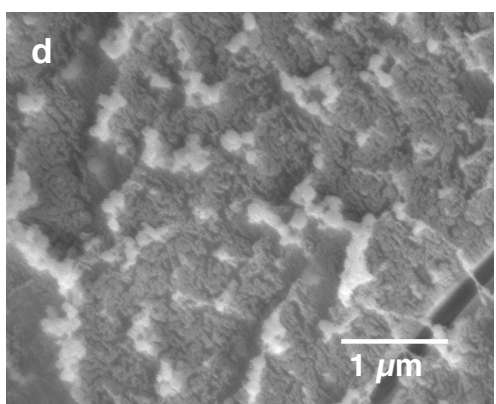

Figure 1. (a) Schematic of nanoparticles used in this study. Left: core-shell nanocapsules, middle: fractal multicore PFCE-PLGA NPs. (b) Chemical structures of components of nanoparticles. Top row lipophilic chelates Gd_01 and Gd_02, and hydrophilic gadoteridol (Prohance). Bottom row: PFCE, PLGA and poly(vinyl alcohol) (PVA) that was used as a surfactant. (c, d) cryogenic Scanning Electron Micrograph (cryoSEM) micrographs of nanoparticles (c) and nanocapsules (d). See also Figure S1 and S2 for larger images. $c=10 \mathrm{mg} \mathrm{mL}^{-1}$ in water. Scale bar $1 \mu \mathrm{m}$.

When lipophilic chelates were used for the synthesis, the concentration of encapsulated gadolinium increased in both fractal multicore and core-shell particles with the added amount chelate, as shown by inductively coupled plasma-mass spectrometry (ICP-MS, Figure 2a, b). The encapsulation of Gd_02 that has a longer hydrophobic chain was slightly higher compared to Gd_01. In contrast, the encapsulation of hydrophilic gadoteridol was very low, despite using a nearly 50-fold higher Gd-concentration than the lipophilic chelates. PLGA is a hydrophobic polymer; consequently, it is very well suited for the encapsulation of hydrophobic cargo, such as lipophilic chelates. Conversely, the hydrophilic compounds, such as gadoteridol, typically tend to remain in the aqueous phase of the emulsion during the miniemulsion process. Thus, it is possible 
to tune only the concentration of the hydrophobic gadolinium chelates during the miniemulsion formulation.
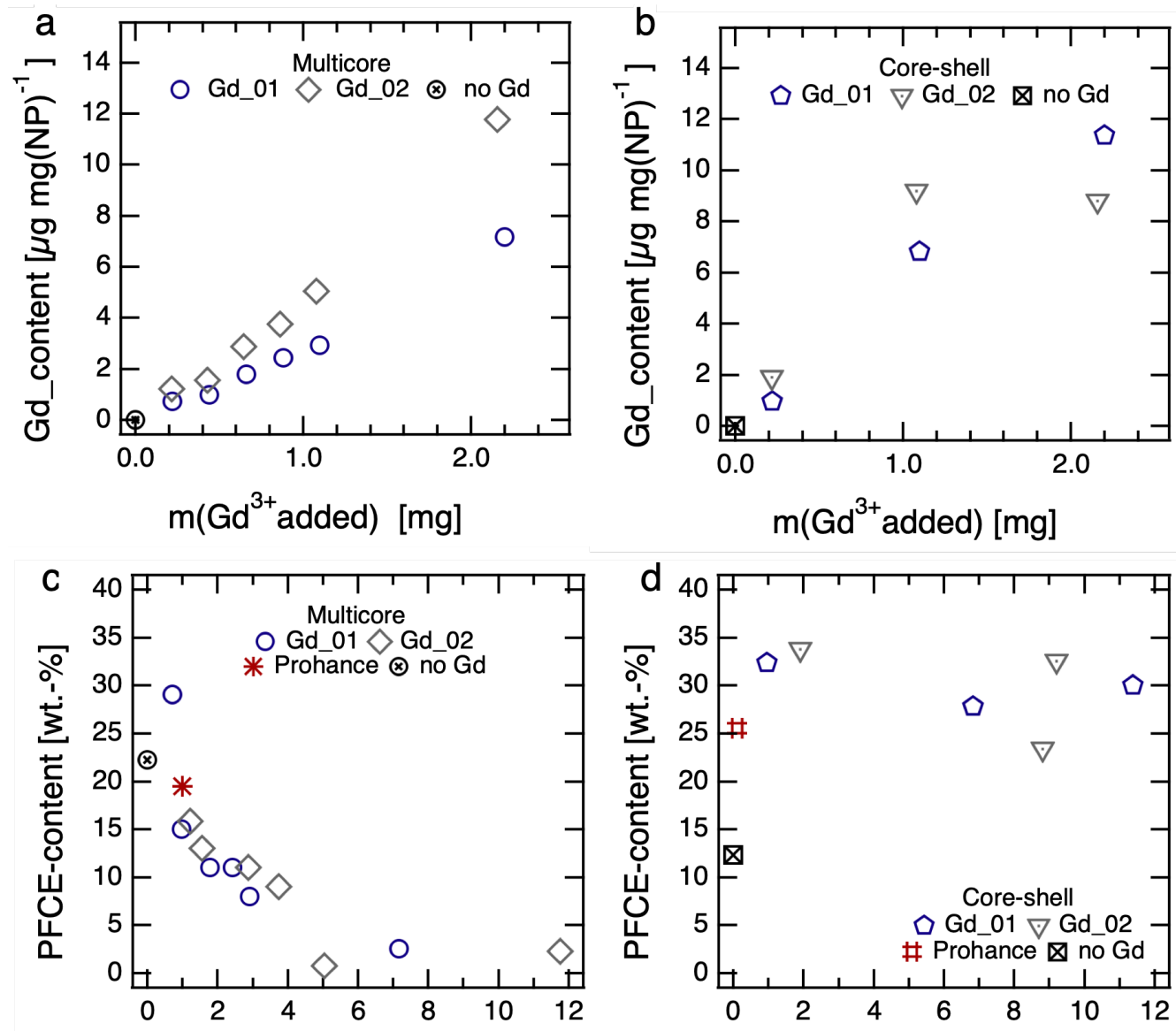

Gd-content $\left[\mu \mathrm{g}(\mathrm{Gd}) \mathrm{mg}(\mathrm{NP})^{-1}\right]$

Gd-content $\left[\mu \mathrm{g}(\mathrm{Gd}) \mathrm{mg}(\mathrm{NP})^{-1}\right]$

Figure 2. Characterization of PFCE-PLGA-NPs and core-shell PFCE-PLGA-capsules loaded with different gadolinium chelates. $(a, b)$ Gadolinium-content versus mass $m$ of gadolinium used for preparation of nanoparticles determined by ICP-MS: (a) fractal multicore nanoparticles, (b) core-shell nanocapsules. When lipophilic gadolinium chelates are used, gadolinium content in nanoparticles increases with increased amount of gadolinium chelate added during the emulsification. In contrast, the encapsulation of Prohance was lower with $0.10 \mu \mathrm{g} \mathrm{mg(NP)^{-1 }}$ in multicore nanoparticles and $0.11 \mu \mathrm{g} \mathrm{mg(NP)^{-1 }}$ in capsules despite that $140 \mathrm{mg}$ Gd were used for encapsulation (not shown for clarity). (c, d) PFCE-content determined by ${ }^{19} \mathrm{~F}$ NMR spectroscopy (trifluoroacetic acid as an internal reference, $\mathrm{D}_{2} \mathrm{O}, 378 \mathrm{MHz}$ ). In multicore nanoparticles (c), the encapsulation of PFCE seem to decrease with an increasing loading of gadolinium chelates. In contrast, the PFCE content in nanocapsules (d) remains constant with an increasing Gd-content. See Table S3 and S4. 
The encapsulation of PFCE showed different trends in fractal multicore nanoparticles and coreshell nanocapsules, as shown by a quantitative ${ }^{19} \mathrm{~F}$ NMR spectroscopy (Figure 2c, d). Thus, the PFCE-content in multicore nanoparticles appears to decrease with increasing concentration of Gd(III) (Figure 2c). This trend was not observed in nanocapsules. Here, the content was even higher compared to capsules that did not contain gadolinium chelate. (Figure $2 c, d$ ). However, it is important to note that the encapsulation of paramagnetic chelates can have different effects on the MR properties in both types of particles. Specifically, the line broadening can influence the quantification, as discussed in the next section in the context of the relaxation times.

Finally, all nanoparticles and nanocapsules did not show any toxic effects, as demonstrated by a standard viability assay using the same dose as typically applied for labeling the cells for ${ }^{19} \mathrm{~F}$ MRI with multicore nanoparticles ${ }^{27}, 30$ ((3-(4,5-dimethylthiazol-2-yl)-2,5-diphenyltetrazolium bromide (MTT)-assay RAW macrophages, Figure S3). The viability values were slightly higher compared to a live cell control. This behavior might originate from an increased phagocytic activity of the cells in presence of nanoparticles and was already observed with other cell types using PFCE-PLGA-NPs without gadolinium chelates. ${ }^{30}$ Hence, the encapsulation of gadolinium chelates did not affect the toxicity of nanoparticles, as was expected because $\mathrm{Gd}$ chelates are used in clinical imaging.

\section{Confinement of PFC in fractal multicore nanoparticles enables the modulation of}

\section{fluorine-19 and proton MR signal.}

After the production and extensive characterization of gadolinium-loaded nanoparticles and nanocapsules, our next step was to study if the encapsulation affected the MR properties of ${ }^{19} \mathrm{~F}$ nucleus. To compare the ${ }^{19} \mathrm{~F}$ relaxation in fractal multicore versus core-shell systems, we measured the ${ }^{19} \mathrm{~F}$ relaxation times by NMR spectroscopy. The relaxation times without gadolinium chelates were similar to earlier studies. ${ }^{35}$ The effects of the gadolinium encapsulation on the 
longitudinal and transverse relaxation rates $R_{1}$ and $R_{2}$ (the inverse relaxation times are shown in Figure 3 (see also Tables S5-S8).

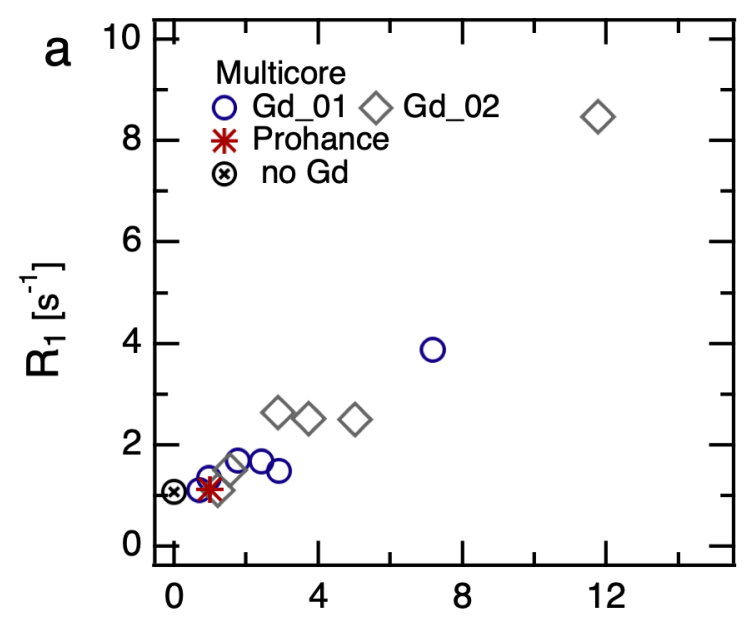

Gd-content $\left[\mu \mathrm{g}(\mathrm{Gd}) \mathrm{mg}(\mathrm{NP})^{-1}\right]$

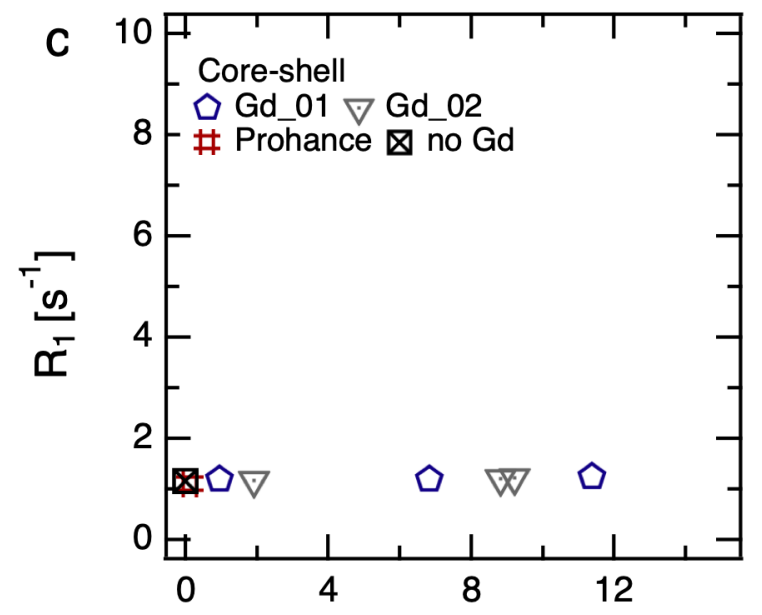

Gd-content $\left[\mu \mathrm{g}(\mathrm{Gd}) \mathrm{mg}(\mathrm{NP})^{-1}\right]$

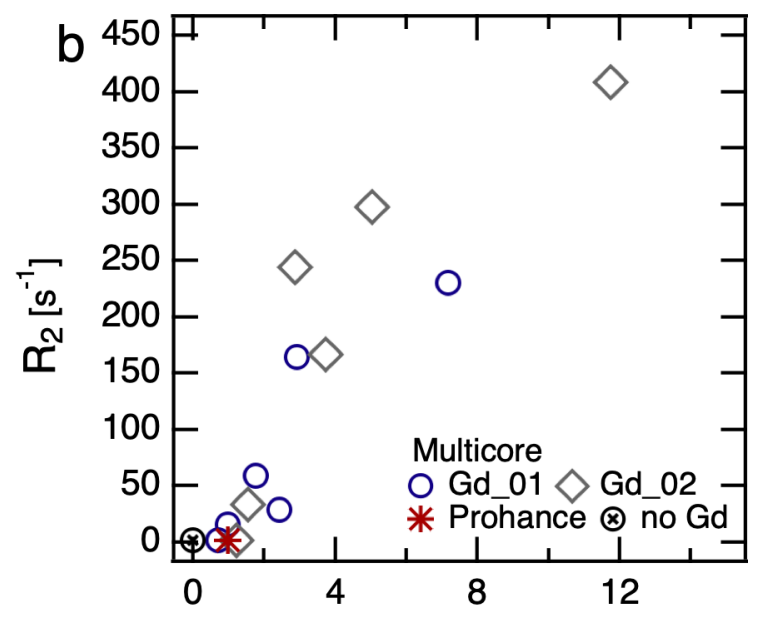

Gd-content $\left[\mu \mathrm{g}(\mathrm{Gd}) \mathrm{mg}(\mathrm{NP})^{-1}\right]$

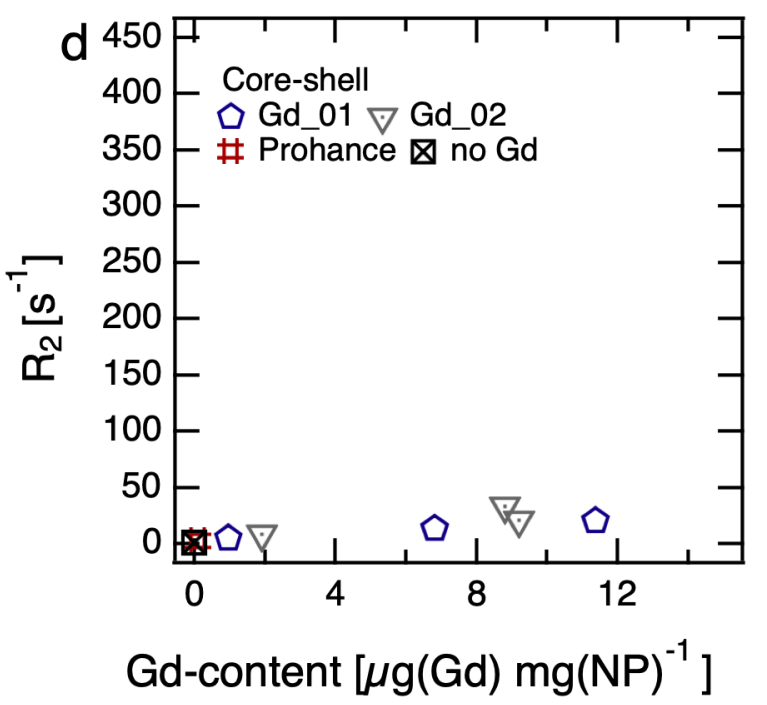

Figure 3. ${ }^{19} \mathrm{~F}$ MR relaxation properties are different in fractal multicore nanoparticles loaded with paramagnetic Gd-chelates compared with core-shell capsules. Longitudinal relaxation rates $R_{1}$ of multicore nanoparticles (a) and core-shell nanocapsules (b). Transverse relaxation rates $\mathbf{R}_{\mathbf{2}}$ of multicore nanoparticles (c) and core-shell nanocapsules (d). Both longitudinal and transverse relaxation rate increased in multicore particles. In core shell capsules the effects were lower due to longer distances between PFCE and gadolinium chelates in both types of particles. Nanoparticles in deuterium oxide, $378 \mathrm{MHz}$. 
The NMR measurements show that the internal structure of nanoparticles strongly affects the relaxation properties of ${ }^{19} \mathrm{~F}$ nucleus (Figure 3 and Tables S5-S8). In multicore nanoparticles, the longitudinal relaxation rate increased almost 8-fold at the highest Gd-loading compared to Gd-free nanoparticles. Moreover, the transverse relaxation was strongly affected and showed approximately 255 -fold increase. In the core-shell nanocapsules, the transverse relaxation rate $\mathrm{R}_{2}$ also increased. However, with only a 2 -fold decrease this effect was much less pronounced compared to multicore nanoparticles. Similarly, the encapsulation of gadolinium chelates showed a small longitudinal relaxation $\mathrm{R}_{1}$ enhancement in the core-shell capsules.

The stark increase of the transverse relaxation rate in multicore nanoparticles can cause the differences in the quantification of PFCE between fractal multicore nanoparticles and core-shell nanocapsules (compare Figure $2 \mathrm{c}$ and $\mathrm{d}$ ). A shorter transverse relaxation time $\mathrm{T}_{2}$ always leads to line broadening of the NMR signal (see Figures S4 and S5). Consequently, it becomes difficult to accurately integrate extraordinarily broad peaks due to the large volume within the baseline. The higher the concentration of encapsulated gadolinium is, the faster the transverse relaxation rate becomes, the broader the PFCE peak appears, and the lower the concentration of PFCE in multicore nanoparticles is detected as a result. Correspondingly, the detected concentration of PFCE was affected by the encapsulation of gadolinium chelates in nanoparticles, but not in the core-shell nanocapsules.

What is the reason behind the altered relaxation properties? The effect of paramagnetic ions on the relaxation times of ${ }^{19} \mathrm{~F}$ nucleus depends on the distance between the ion and the ${ }^{19} \mathrm{~F}$ nucleus. ${ }^{14,23,24}$ Previously, we reported the structural characterization of gadolinium chelate-free and gadoteridol-loaded fractal multicore nanoparticles and core-shell nanocapsules by the SmallAngle Neutron Scattering (SANS). ${ }^{26}$ Note that the radii obtained with SANS are typically smaller than the hydrodynamic sizes from the DLS, as reported previously. ${ }^{26}$ In multicore nanoparticles, each PFCE core typically displays a radius of $9-12 \mathrm{~nm}$ and a shell thickness of $4 \mathrm{~nm}$, with some 
batch-to-batch variation. ${ }^{26}$ The core-shell capsules usually display a core radius of around $20 \mathrm{~nm}$ that is surrounded a shell of a similar thickness. ${ }^{26}$

The distance between gadolinium and PFCE is shorter in fractal multicore nanoparticles compared to nanocapsules, if we assume that gadolinium chelates are distributed homogenously within the PLGA matrix. Thus, the farthest possible distance between PFCE molecule that is located in the center of the core and the gadolinium chelate in outer part of the shell is approximately $15 \mathrm{~nm}$. The distance between PFCE in the center or the core and gadolinium chelate in the inner part of the shell is $9-12 \mathrm{~nm}$. This distance decreases the closer a PFCE molecule is located to the shell. For the capsules, the farthest distance is $40 \mathrm{~nm}$ and the distance between PFCE in the center and gadolinium molecule in the inner part of the shell is approximately $20 \mathrm{~nm}$. Thus, this distance is almost twice as high compared to the distance in the multicore nanoparticles. However, PRE typically decreases with the distance ${ }^{6}{ }^{6}{ }^{10,14,16,17}$ Hence, shortening the distance alone would not be sufficient to modulate the PFCE molecules located in the center of the core. Thus, the diffusion of PFCE and its confinement in the fractal multicore structure appear to affect the PRE.

Indeed, other reports also suggested that diffusion of PFCE can influence the PRE. Particularly, several reports showed that conjugation of gadolinium chelates to the surface of PFCE-loaded silica capsules results in the enhancement of transverse relaxation rate. ${ }^{36-38}$ The authors did not report the PRE of the longitudinal relaxation rate. Importantly, other groups did not observe PRE of longitudinal relaxation ${ }^{19} \mathrm{~F}$ upon the incorporation gadolinium in the surfactant layer of emulsions. ${ }^{12,23,24}$ Similarly, in this study we detected only moderate effects on PRE in the control core-shell nanocapsules. Thus, the formation of confining fractal multicore structure appears to further affect the PRE of the longitudinal relaxation rate. Typically, the diffusion speed of confined liquids is reduced compared to bulk. ${ }^{39}$ However, liquid PFCs display a very low intermolecular cohesiveness, ${ }^{40}$ which may countervail these effects. Additionally, the distance that a PFCE 
molecule has to diffuse to relocate from the core center to the shell becomes lower due to the formation the confining fractal structure. As a result, the PRE of both longitudinal and transverse relaxation can be observed.

Lastly, the concentration of gadolinium affects the PRE. When the concentration of the gadolinium chelate increases within the polymeric matrix, it automatically leads to an increased number of gadolinium nuclei in the inner parts of the PLGA-shell that are closer to PFCE-phase. Therefore, an additional increase of both longitudinal and transverse relaxation rates can be observed in fractal multicore nanoparticles with increasing concentration of encapsulated gadolinium chelate. Overall, our data shows that the structural properties of nanoparticles affect the paramagnetic relaxation of ${ }^{19} \mathrm{~F}$ nucleus.

Finally, ${ }^{1} \mathrm{H} /{ }^{19} \mathrm{~F} \mathrm{MRI}$ shown that the encapsulation of paramagnetic chelates affected not only the fluorine, but also the proton signal in fractal multicore particles (Figure 4a). Thus, the ${ }^{19} \mathrm{~F}$ signal intensity decreased with increasing concentration of gadolinium, as expected due to the enhanced transversal relaxation rate (Figure $4 \mathrm{a}$, see Table S9 for the signal-to-noise values). In contrast, the proton signal increased with increasing encapsulation of chelates. The signal-to-noise-ratio of proton signal increased from 17 to 49 , when the gadolinium content increased from 0 to $2.4 \mu \mathrm{g}(\mathrm{Gd})$ $\times \mathrm{mg}(\mathrm{NPs})^{-1}$. In fractal multicore particles, gadolinium is encapsulated within the PLGA matrix. The PLGA matrix is hydrated and remains permeable to water and other small molecules, ${ }^{26}$ enabling to modulate the proton signal. 


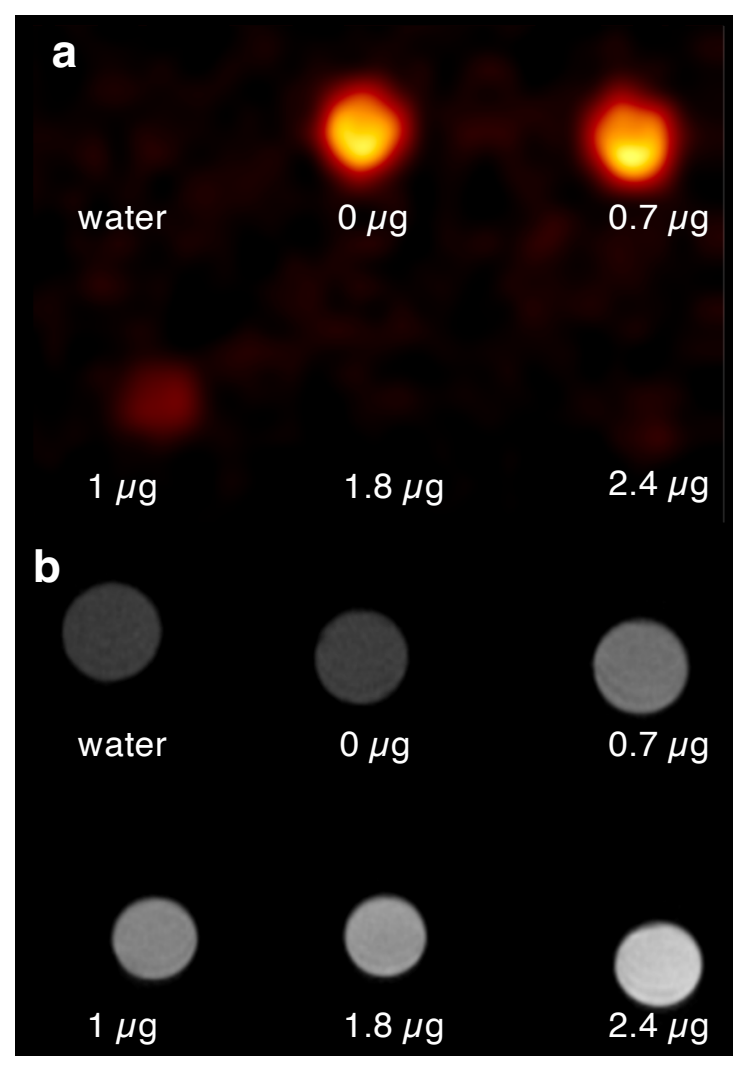

Figure 4. ${ }^{19} \mathrm{~F}$ (a) and ${ }^{1} \mathrm{H}$ (b) MRI of fractal multicore nanoparticles that contain a different amount of gadolinium chelates. The gadolinium content in $\mu \mathrm{g}$ per $\mathrm{mg}$ of nanoparticles is indicated under each sample; nanoparticles are dispersed in water at a concentration of 10 $\mathrm{mg} \mathrm{mL}^{-1}$. Water and nanoparticles loaded only with PFCE were used as controls. The proton signal is increasing and fluorine signal decreasing with an increasing Gd-content. Thus, it is possible to alter the signal of both nuclei, with changes relevant for imaging, when using fractal multicore PLGA nanoparticles for the encapsulation. ${ }^{1} \mathrm{H}$ MRI acquired with $\mathrm{T}_{1^{-}}$ weighted 2D fast low angle shot (FLASH) sequence and ${ }^{19} \mathrm{~F}$ MRI with 3D RARE sequence, $11.7 \mathrm{~T}$.

Thus, the encapsulation of paramagnetic chelates in confining multicore nanoparticles enables to modulate longitudinal and transverse relaxation rates of ${ }^{19} \mathrm{~F}$-nucleus, along with proton signal from the aqueous environment of nanoparticles. In the emulsions, it is only to efficiently modulate either the proton or the fluorine relaxation, but not both simultaneously. The ${ }^{1} \mathrm{H}$ relaxation is altered, when the paramagnetic chelate is attached to the shell. However, in this case, the PRE ${ }^{19} \mathrm{~F}$ nuclei in the fluorous phase is minor. ${ }^{23}$ Conversely, the fluorophilic chelates are dispersed in the PFC phase, shortening the ${ }^{19} \mathrm{~F}$ relaxation times, but remain inaccessible to water. ${ }^{14}$ Thus, only 
the PRE of ${ }^{19} \mathrm{~F}$-signal is obtained. Differently to emulsions, co-encapsulation of PFC and gadolinium chelates in fractal multicore nanoparticles enables to modulate both ${ }^{19} \mathrm{~F}$ and ${ }^{1} \mathrm{H}$ signal. Here we used gadolinium-(III) chelates, which are known to cause a strong PRE of transverse relaxation. In the future studies these effects could be reduced by using other paramagnetic ions, similarly as Ahrens' group reported for fluorophilic chelates. ${ }^{13,}{ }^{15}$ These findings can be used for development of dual-responsivity probes in the future.

\section{Conclusion}

A co-encapsulation of PFC with paramagnetic chelates in nanoparticles for ${ }^{19} \mathrm{~F} \mathrm{MRI}$ can lead to multifunctional imaging probes that show high potential to be applied in high-sensitive ${ }^{19} \mathrm{~F} \mathrm{MRI}$, or in sensing applications. Yet, it remained not possible to modulate both the ${ }^{19} \mathrm{~F}$-signal of PFC inside the nanoparticles and the proton signal from the aqueous environment of the probe. Here we studied the influence on the internal structure of nanoparticles on the PRE and compared liquid PFC-loaded PLGA nanoparticles with fractal multicore structure versus core-shell structure. In both systems PFC forms the phase-separated core(s); hydrophobic gadolinium chelates are loaded in the PLGA matrix. Our results show that the structural properties play an important role for ${ }^{19} \mathrm{~F}$ MR relaxation. The effects of the paramagnetic chelate on the longitudinal relaxation of PFC were minor in core-shell nanoparticles. Only an increase of the transverse relaxation rate could be detected. Interestingly, in fractal multicore nanoparticles both longitudinal and transverse relaxation rates of ${ }^{19} \mathrm{~F}$ nucleus increased with increasing concentration of paramagnetic chelate. Moreover, the encapsulation of paramagnetic chelates in fractal nanoparticles enables to modulate the signal not only of ${ }^{19} \mathrm{~F}$ nucleus but also of proton. In the future, the results of our study can be applied to simultaneously tune the ${ }^{19} \mathrm{~F}$ and ${ }^{1} \mathrm{H}$ MR features of agents that contain a liquid PFC and paramagnetic chelates, for example, in production of high-sensitivity probes that can 
additionally enhance proton signal, in sensing applications and for design the on-/off-probes that change their signal intensity upon degradation of nanomaterials.

\section{Funding Sources}

The authors acknowledge the funding from ERC-2014-StG-336454-CoNQUeST, TTW-NWO open technology grant STW-14716, ERC-2015-PoC-713524-CONQUEST, ERC-2019-PoC862989-CENYA, and ERA-CVD JTC2017-044.

\section{Acknowledgements}

The authors kindly thank Bijal K. Bahuleyan (Univ. Rheims) Paul van der Ven (RU), Geert-Jan Janssen $(R U)$ and the General Instruments Department $(R U)$, Peter van Dijk (RU), Daniela Wilson $(\mathrm{RU})$, Eric van Dinther (Radboudumc), Oya Tagit (Radboudumc), Massis Krekorian (Radboudumc), I. Jolanda M. de Vries (Radboudumc) and Carl G. Figdor (Radboudumc).

\section{Corresponding authors:}

*mangala.srinivas@wur.nl

*o.koshkina@utwente.nl

\section{Author contributions:}

‡MV and PBW contributed equally

‡OK and MS contributed equally

\section{Materials and Methods}

\section{Materials}


All chemicals were used as received: PLGA (Resomer 502H) from Evonik Industries, Germany, dichloromethane (DCM) (EMSURE, Merck Millipore, Darmstadt, Germany), Polyvinyl alcohol (PVA), deuterium oxide and trifluoroacetic acid (TFA) were purchased from Sigma-Aldrich (St. Louis, MO, USA), perfluoro-15-crown-5 ether (PFCE) (Exfluor, Texas, United States), Prohance (Bracco, Amsterdam, Netherlands). Lipophilic gadolinium chelates were synthesized as described previously. ${ }^{34}$ Water was purified by a Synergy water purification system from Merck.

\section{Methods}

Synthesis of multicore nanoparticles. PVA $(0.5 \mathrm{~g})$ was dissolved in ultrapure water $(25 \mathrm{~g})$ under magnetic stirring. PLGA $(0.1 \mathrm{~g})$ was dissolved in dichloromethane $(3 \mathrm{~mL})$ and mixed with PFCE $(0.9 \mathrm{~mL})$ by pipetting it up and down with a glass pipette. In parallel, stock solutions of Gd-chelates

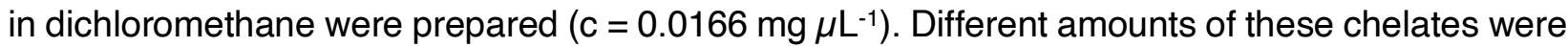
added to the organic phase. For preparation of particles with gadoteridol, Prohance $(1.78 \mathrm{~mL}$, corresponds to $497 \mathrm{mg}$ gadoteridol and $140 \mathrm{mg} \mathrm{Gd}$ ) were added to the organic phase and sonicated for $15 \mathrm{~s}$. Afterwards, the sonication of the aqueous surfactant solution was started, and the organic phase was added rapidly with a glass pipette (microtip Branson digital Sonifier s250, Missouri, United States). The miniemulsion was sonicated on an ice-water bath for 3 minutes at $40 \%$ amplitude. As PFCE is both hydrophobic and lipophobic the organic phase has to be constantly premixed by pipetting during the addition to ensure that PFCE and PLGA phases are added simultaneously. After sonication, DCM was evaporated overnight at room temperature under stirring to achieve solidification of the particles. The dispersions were transferred to $50 \mathrm{~mL}$ tubes and washed four times by centrifugation at $16000 \mathrm{~g}\left(4^{\circ} \mathrm{C}, 35 \mathrm{~min}\right)$ and supernatant replacement. After the second washing step, the samples were sonicated in ultrasonic bath (sonication bath, Diagenode Bioruptor, Seraign, Belgium) and the washing cycle was repeated. After washing, particles were resuspended in water $(5 \mathrm{~mL})$, frozen with liquid nitrogen and freezedried. The resulting powder was stored at $-20^{\circ} \mathrm{C}$. 
Synthesis of nanocapsules. For synthesis of nanocapsules sodium cholate $(0.375 \mathrm{~g})$ that was used as a surfactant for sonication step was dissolved in water (24.625 g, 1.5 wt.-\% solution). PLGA $(0.1 \mathrm{~g})$ was dissolved in dichloromethane $(2 \mathrm{~mL})$ and mixed with PFCE $(0.9 \mathrm{~mL})$ by pipetting it up and down with a glass pipette. Different amounts of these chelates were added to the organic phase (corresponding to $1 \mathrm{mg}, 5 \mathrm{mg}$ and $10 \mathrm{mg}$ of chelate). Some samples were prepared with the addition of Prohance $(1.78 \mathrm{~mL})$. The organic phase was then added with a glass pipette to the sodium cholate solution. As PFCE is both hydrophobic and lipophobic the organic phase had to be premixed before sonication to ensure that PFCE and PLGA phases are added simultaneously. The miniemulsion was sonicated in an ice bath for 3 minutes at $40 \%$ amplitude (microtip Branson digital sonifier s250, Missouri, United States). After sonication, DCM was evaporated overnight at room temperature under stirring to achieve solidification of the particles. To exchange the surfactant, $10 \mathrm{~g}$ of PVA solution (1.96 wt.-\%) was added to the suspension, followed by the continuous stirring at $4{ }^{\circ} \mathrm{C}$ for 4 days. After 4 days, the capsules were washed 4 times with deionized water through centrifugation $\left(16098 \mathrm{~g}, 35\right.$ minutes, $\left.4^{\circ} \mathrm{C}\right)$, resuspended in $5 \mathrm{~mL}$ of water, frozen (liquid $\mathrm{N}_{2}$ ) and freeze-dried. The nanocapsules in a powder form were stored at $-20{ }^{\circ} \mathrm{C}$.

Inductively coupled plasma-mass-spectrometry (ICP-MS) to measure Gd-content was determined by ICP-MS X series I with quadrupole Mass Spectrometer. Samples were prepared by adding $10 \mathrm{mg}$ of the nanoparticles to $145 \mu \mathrm{L} \mathrm{HNO}$ (1\%) and left overnight at room temperature in order to let the particles fully degrade. Afterwards, the samples were filled up to a total volume of $10 \mathrm{~mL}$ prior the measurement.

Dynamic light scattering (DLS) to determine the particle size and the polydispersity index (PDI) was done on Malvern Zetasizer ZS nano instrument. The particles were dispersed in ultrapure water at a concentration of $0.01 \mathrm{mg} \mathrm{mL}^{-1}$.

${ }^{19} \mathrm{~F}$ NMR was measured with Bruker Avance III $400 \mathrm{MHz}$ spectrometer equipped with a $5 \mathrm{~mm}$ $\mathrm{BBFO}+$ probe at $298 \mathrm{~K}$. 
Determination of PFCE content was done by quantitative ${ }^{19} \mathrm{~F}$ NMR. The nanoparticles and capsules were resuspended with $400 \mu \mathrm{L} \mathrm{D}_{2} \mathrm{O}$ and $100 \mu \mathrm{L} 0.1$ vol.-\% trifluoroacetic acid (TFA) as an internal reference. The interscan delay d1 was set to $5 \times \mathrm{T}_{1}$.

For relaxation time measurements approximately $5 \mathrm{mg}$ of particles were resuspended in $\mathrm{D}_{2} \mathrm{O}$ and transferred to an NMR tube. The ${ }^{19} \mathrm{~F} 90^{\circ}$ pulse was calibrated prior the measurement.

$T_{1}$ measurements were done using the inversion recovery method of a $180^{\circ}$ pulse followed by a variable delay period for transverse magnetization to relax longitudinally followed by a $90^{\circ}$ detection pulse. The $T_{1}$ was first estimated using a simple 1D sequence and then a full pseudo2D acquisition was acquired with a list of delays with an interscan delay set to $5 \times \mathrm{T}_{1}$, slowest. The recovery was fit to an exponential growth function which revealed the $T_{1}$. The ${ }^{19} \mathrm{~F} T_{1}$ experiments were performed without ${ }^{1} \mathrm{H}$ decoupling.

$\mathrm{T}_{2}$ Measurements were performed using the CPMG method of a $90^{\circ}$ pulse followed by a train of tau-180-tau, where tau is a variable delay. For rapid $T_{2}$-relaxing nuclei ( $T_{2}<30 \mathrm{~ms}$ ), a tau of 0.5 ms was used. For other, slower $T_{2}$-relaxing nuclei, a tau of $1.2 \mathrm{~ms}$ was used. The $T_{2}$ was first estimated using a simple 1D sequence. Afterwards, a full pseudo-2D acquisition was acquired with a list of refocusing pulse repetitions ranging from 2 to 1750 and an interscan delay set to $5 \mathrm{x}$ $\mathrm{T}_{1}$, slowest. The recovery was fit to a mono-exponential decay function which revealed the $T_{2}$.

Cryogenic Scanning Electron Microscopy (CryoSEM) images were obtained with JEOL 6300F Field Emission Scanning Electron Microscope. The samples (8 $\left.\mu \mathrm{L}, 10 \mathrm{mg} \mathrm{mL}^{-1}\right)$ were pipetted in 2 rivets that are placed together. Then the sample is frozen in liquid nitrogen slush and with a cryo transfer device placed in a Oxford Alto 2500 cryo-station. There the top rivet is broken and the sample is heated to $-95^{\circ} \mathrm{C}$ for 5 minutes. Then a coating of $60 / 40 \mathrm{Au} / \mathrm{Pd}$ is done and the sample is transferred to the cryoSEM.

${ }^{1} \mathrm{H}$ and ${ }^{19} \mathrm{~F}$ Magnetic Resonance Imaging was done at Brucker Biospec 11.7 T. The samples with gadolinium content of $0,0.7,0.9,1.8$ and $2.5 \mu \mathrm{g} \mathrm{mg}^{-1}$. The concentration of nanoparticles was 
$10 \mathrm{mg} \mathrm{mL}^{-1}$, which is also often use for in vivo imaging. ${ }^{1} \mathrm{H}$ MRI was acquired with $\mathrm{T}_{1}$-weighted $2 \mathrm{D}$ fast low angle shot (FLASH) sequence: TR $104 \mathrm{~ms}$, TE $2.25 \mathrm{~ms}$, flip angle $40^{\circ}, 2$ averages, matrix $256 \times 256$, Field of View (FOV) $32 \times 32 \mathrm{~mm}, 12$ slices with a slice thickness of $1 \mathrm{~mm}$, scan time $53 \mathrm{~s} .{ }^{19} \mathrm{~F} \mathrm{MRI}$ was measured with a 3D RARE sequence with the following parameters: TR $1 \mathrm{~s}$, TE $6.5 \mathrm{~ms}$, turbo factor 8, averages, matrix $32 \times 32 \times 12$, FOV $32 \times 32 \times 24 \mathrm{~mm}$, scan time 6:20 $\min$.

\section{References}

1. Tirotta, I.; Dichiarante, V.; Pigliacelli, C.; Cavallo, G.; Terraneo, G.; Bombelli, F. B.; Metrangolo, P.; Resnati, G., F-19 Magnetic Resonance Imaging (MRI): From Design of Materials to Clinical Applications. Chem. Rev. (Washington, DC, U. S.) 2015, 115 (2), 1106-1129.

2. Ruiz-Cabello, J.; Barnett, B. P.; Bottomley, P. A.; Bulte, J. W., Fluorine (19F) MRS and MRI in biomedicine. NMR Biomed. 2011, 24 (2), 114-129.

3. Chapelin, F.; Capitini, C. M.; Ahrens, E. T., Fluorine-19 MRI for detection and quantification of immune cell therapy for cancer. J. Immunother. Cancer 2018, 6, 11.

4. Janjic, J. M.; Ahrens, E. T., Fluorine-containing nanoemulsions for MRI cell tracking. WIRES Nanomed. Nanobiotechnol. 2009, 1 (5), 492-501.

5. Pisani, E.; Tsapis, N.; Galaz, B.; Santin, M.; Berti, R.; Taulier, N.; Kurtisovski, E.; Lucidarme, O.; Ourevitch, M.; Doan, B. T.; Beloeil, J. C.; Gillet, B.; Urbach, W.; Bridal, S. L.; Fattal, E., Perfluorooctyl Bromide Polymeric Capsules as Dual Contrast Agents for Ultrasonography and Magnetic Resonance Imaging. Adv. Funct. Mater. 2008, 18 (19), 2963-2971.

6. Zhang, C.; Liu, T.; Wang, W.; Bell, C. A.; Han, Y.; Fu, C.; Peng, H.; Tan, X.; Král, P.; Gaus, K.; Gooding, J. J.; Whittaker, A. K., Tuning of the Aggregation Behavior of Fluorinated Polymeric Nanoparticles for Improved Therapeutic Efficacy. ACS Nano 2020, 14 (6), 7425-7434.

7. $\quad$ Boissenot, T.; Fattal, E.; Bordat, A.; Houvenagel, S.; Valette, J.; Chacun, H.; Gueutin, C.; Tsapis, N., Paclitaxel-loaded PEGylated nanocapsules of perfluorooctyl bromide as theranostic agents. Eur. J. Pharm. Biopharm. 2016, 108, 136-144.

8. Lin, H.; Tang, X.; Li, A.; Gao, J., Activatable 19F MRI Nanoprobes for Visualization of Biological Targets in Living Subjects. Adv. Mater. (Weinheim, Ger.) n/a (n/a), 2005657.

9. Wang, C.; Adams, S. R.; Ahrens, E. T., Emergent Fluorous Molecules and Their Uses in Molecular Imaging. Acc. Chem. Res. 2021.

10. Xie, D.; Yu, M.; Kadakia, R. T.; Que, E. L., 19F Magnetic Resonance Activity-Based Sensing Using Paramagnetic Metals. Acc. Chem. Res. 2020, 53 (1), 2-10.

11. Gambino, G.; Gambino, T.; Pohmann, R.; Angelovski, G., A ratiometric F-19 MR-based method for the quantification of $\mathrm{Ca} 2+$ using responsive paramagnetic probes. Chem. Commun. (Cambridge, U. K.) 2020, 56 (24), 3492-3495.

12. Gambino, G.; Gambino, T.; Angelovski, G., Combination of Bioresponsive Chelates and Perfluorinated Lipid Nanoparticles Enables in vivo MRI Probe Quantification. Chem. Commun. (Cambridge, U. K.) 2020.

13. Jahromi, A. H.; Wang, C.; Adams, S. R.; Zhu, W.; Narsinh, K.; Xu, H.; Gray, D. L.; Tsien, R. Y.; Ahrens, E. T., Fluorous-Soluble Metal Chelate for Sensitive Fluorine-19 Magnetic Resonance Imaging Nanoemulsion Probes. ACS Nano 2019, 13 (1), 143-151. 
14. Kislukhin, A. A.; Xu, H. Y.; Adams, S. R.; Narsinh, K. H.; Tsien, R. Y.; Ahrens, E. T., Paramagnetic fluorinated nanoemulsions for sensitive cellular fluorine-19 magnetic resonance imaging. Nat. Mater. 2016, 15 (6), 662-+.

15. Rho, J.; Stares, E.; Adams, S. R.; Lister, D.; Leach, B.; Ahrens, E. T., Paramagnetic Fluorinated Nanoemulsions for in vivo F-19 MRI. Mol. Imaging. Biol. 2020, 22 (3), 665-674.

16. Peterson, K. L.; Srivastava, K.; Pierre, V. C., Fluorinated Paramagnetic Complexes: Sensitive and Responsive Probes for Magnetic Resonance Spectroscopy and Imaging. Front. Chem. 2018, 6 (160).

17. Clore, G. M.; Iwahara, J., Theory, Practice, and Applications of Paramagnetic Relaxation Enhancement for the Characterization of Transient Low-Population States of Biological Macromolecules and Their Complexes. Chem. Rev. (Washington, DC, U. S.) 2009, 109 (9), 41084139.

18. Riess, J. G., Fluorous micro-and nanophases with a biomedical perspective. Tetrahedron 2002, 58 (20), 4113-4131.

19. Cavallo, G.; Metrangolo, P.; Milani, R.; Pilati, T.; Priimagi, A.; Resnati, G.; Terraneo, G., The Halogen Bond. Chem. Rev. (Washington, DC, U. S.) 2016, 116 (4), 2478-2601.

20. Riess, J. G., Understanding the Fundamentals of Perfluorocarbons and Perfluorocarbon Emulsions Relevant to In Vivo Oxygen Delivery. Artif. Cells, Blood Substitutes, Biotechnol. 2005, 33 (1), 47-63.

21. Matsushita, H.; Mizukami, S.; Sugihara, F.; Nakanishi, Y.; Yoshioka, Y.; Kikuchi, K., Multifunctional Core-Shell Silica Nanoparticles for Highly Sensitive 19F Magnetic Resonance Imaging. Angew. Chem. Int. Ed. 2014, 53 (4), 1008-1011.

22. Lee, A. L.; Gee, C. T.; Weegman, B. P.; Einstein, S. A.; Juelfs, A. R.; Ring, H. L.; Hurley, K. R.; Egger, S. M.; Swindlehurst, G.; Garwood, M.; Pomerantz, W. C. K.; Haynes, C. L., Oxygen Sensing with Perfluorocarbon-Loaded Ultraporous Mesostructured Silica Nanoparticles. ACS Nano 2017, 11 (6), 5623-5632.

23. de Vries, A.; Moonen, R.; Yildirim, M.; Langereis, S.; Lamerichs, R.; Pikkemaat, J. A.; Baroni, S.; Terreno, E.; Nicolay, K.; Strijkers, G. J.; Grull, H., Relaxometric studies of gadoliniumfunctionalized perfluorocarbon nanoparticles for MR imaging. Contrast Media Mol. Imaging 2014, 9 (1), 83-91.

24. Neubauer, A. M.; Myerson, J.; Caruthers, S. D.; Hockett, F. D.; Winter, P. M.; Chen, J.; Gaffney, P. J.; Robertson, J. D.; Lanza, G. M.; Wickline, S. A., Gadolinium-modulated 19F signals from perfluorocarbon nanoparticles as a new strategy for molecular imaging. Magn. Reson. Med. 2008, 60 (5), 1066-1072.

25. Peng, Q.; Li, Y.; Bo, S.; Yuan, Y.; Yang, Z.; Chen, S.; Zhou, X.; Jiang, Z.-X., Paramagnetic nanoemulsions with unified signals for sensitive 19F MRI cell tracking. Chem. Commun. (Cambridge, U. K.) 2018, 54 (47), 6000-6003.

26. Koshkina, O.; Lajoinie, G.; Bombelli, F. B.; Swider, E.; Cruz, L. J.; White, P.; Schweins, R.; Dolen, Y.; Dinther, E. v.; Riessen, N. K. v.; Rogers, S. E.; Fokkink, R.; Voets, I. K.; Eck, E. R. H. v.; Heerschap, A.; Versluis, M.; Korte, C. d.; Figdor, C.; Vrie, I. J. M. d.; Srinivas, M., Multicore liquid perfluorocarbon-loaded multimodal nanoparticles for stable ultrasound and 19F MRI applied to in vivo cell tracking. Adv. Funct. Mater. 2019, 29 (19), 1806485.

27. Srinivas, M.; Cruz, L. J.; Bonetto, F.; Heerschap, A.; Figdor, C. G.; De Vries, I. J. M., Customizable, multi-functional fluorocarbon nanoparticles for quantitative in vivo imaging using 19F MRI and optical imaging. Biomaterials 2010, 31 (27), 7070-7077.

28. Jacoby, C.; Temme, S.; Mayenfels, F.; Benoit, N.; Krafft, M. P.; Schubert, R.; Schrader, J.; Flogel, U., Probing different perfluorocarbons for in vivo inflammation imaging by F-19 MRI: image reconstruction, biological half-lives and sensitivity. NMR Biomed. 2014, 27 (3), 261-271.

29. Staal, A. H. J.; Becker, K.; Tagit, O.; Koen van Riessen, N.; Koshkina, O.; Veltien, A.; Bouvain, P.; Cortenbach, K. R. G.; Scheenen, T.; Flögel, U.; Temme, S.; Srinivas, M., In vivo 
clearance of $19 \mathrm{~F} \mathrm{MRI} \mathrm{imaging} \mathrm{nanocarriers} \mathrm{is} \mathrm{strongly} \mathrm{influenced} \mathrm{by} \mathrm{nanoparticle} \mathrm{ultrastructure.}$ Biomaterials 2020, 120307.

30. Hoogendijk, E.; Swider, E.; Staal, A. H. J.; White, P. B.; van Riessen, N. K.; Glaßer, G.; Lieberwirth, I.; Musyanovych, A.; Serra, C. A.; Srinivas, M.; Koshkina, O., Continuous-Flow Production of Perfluorocarbon-Loaded Polymeric Nanoparticles: From the Bench to Clinic. ACS Appl. Mater. Interfaces 2020, 12 (44), 49335-49345.

31. Krekorian, M.; Sandker, G. G. W.; Cortenbach, K. R. G.; Tagit, O.; van Riessen, N. K.; Raavé, R.; Srinivas, M.; Figdor, C. G.; Heskamp, S.; Aarntzen, E. H. J. G., Characterization of Intrinsically Radiolabeled Poly(lactic-co-glycolic acid) Nanoparticles for ex Vivo Autologous Cell Labeling and in Vivo Tracking. Bioconjugate Chem. 2021.

32. Swider, E.; Daoudi, K.; Staal, A. H.; Koshkina, O.; van Riessen, N. K.; van Dinther, E.; de Vries, I. J. M.; de Korte, C. L.; Srinivas, M., Clinically-Applicable Perfluorocarbon-Loaded Nanoparticles For In vivo Photoacoustic, 19F Magnetic Resonance And Fluorescent Imaging. Nanotheranostics 2018, 2 (3), 258-268.

33. Swider, E.; Koshkina, O.; Tel, J.; Cruz, L. J.; de Vries, I. J. M.; Srinivas, M., Customizing poly(lactic-co-glycolic acid) particles for biomedical applications. Acta Biomater. 2018, 73, 38-51.

34. Rigaux, G.; Roullin, V. G.; Cadiou, C.; Portefaix, C.; Van Gulick, L.; Bœuf, G.; Andry, M. C.; Hoeffel, C.; Vander Elst, L.; Laurent, S.; Muller, R.; Molinari, M.; Chuburu, F., A new magnetic resonance imaging contrast agent loaded into poly(lacide-co-glycolide) nanoparticles for longterm detection of tumors. Nanotechnology 2014, 25 (44), 445103.

35. Koshkina, O.; White, P. B.; Staal, A. H. J.; Schweins, R.; Swider, E.; Tirotta, I.; Tinnemans, P.; Fokkink, R.; Veltien, A.; van Riessen, N. K.; van Eck, E. R. H.; Heerschap, A.; Metrangolo, P.; Baldelli Bombelli, F.; Srinivas, M., Nanoparticles for "two color" 19F magnetic resonance imaging: Towards combined imaging of biodistribution and degradation. J. Colloid Interface Sci. 2020, 565, 278-287.

36. Akazawa, K.; Sugihara, F.; Nakamura, T.; Mizukami, S.; Kikuchi, K., Highly Sensitive Detection of Caspase-3/7 Activity in Living Mice Using Enzyme-Responsive 19F MRI Nanoprobes. Bioconjugate Chem. 2018, 29 (5), 1720-1728.

37. Akazawa, K.; Sugihara, F.; Minoshima, M.; Mizukami, S.; Kikuchi, K., Sensing caspase-1 activity using activatable 19F MRI nanoprobes with improved turn-on kinetics. Chem. Commun. (Cambridge, U. K.) 2018, 54 (83), 11785-11788.

38. Nakamura, T.; Matsushita, H.; Sugihara, F.; Yoshioka, Y.; Mizukami, S.; Kikuchi, K., Activatable 19F MRI Nanoparticle Probes for the Detection of Reducing Environments. Angew. Chem. Int. Ed. 2015, 54 (3), 1007-1010.

39. Thompson, W. H., Perspective: Dynamics of confined liquids. J. Chem. Phys. 2018, 149 (17), 170901.

40. Riess, J. G., Fluorous micro- and nanophases with a biomedical perspective. Tetrahedron 2002, 58 (20), 4113-4131. 


\section{TOC graphic}

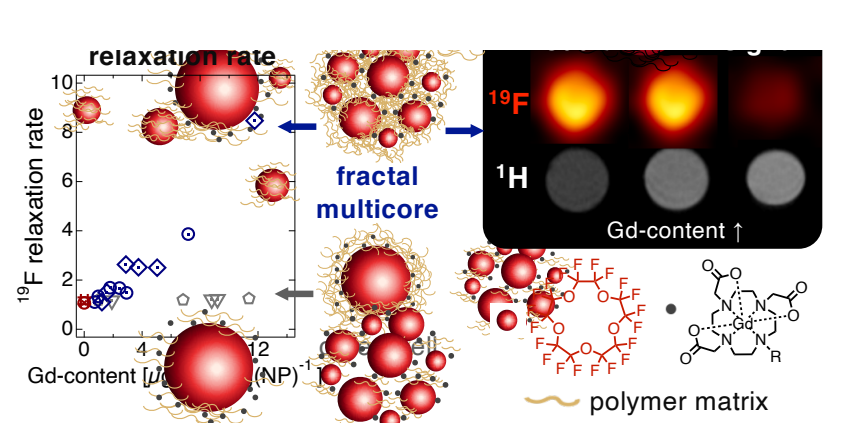

